\title{
Analytical Studies on Holographic Superconductors in Gauss-Bonnet Gravity
}

\author{
Huai-Fan Li ${ }^{1,2,3}$, Rong-Gen Cai ${ }^{2}$, Hai-Qing Zhang ${ }^{2}$ \\ ${ }^{1}$ Department of Physics and Institute of Theoretical Physics, \\ Shanxi Datong University, Datong 037009, China \\ ${ }^{2}$ Key Laboratory of Frontiers in Theoretical Physics, \\ Institute of Theoretical Physics, Chinese Academy of Sciences, \\ P.O. Box 2735, Beijing 100190, China \\ ${ }^{3}$ Department of Applied Physics, Xi' an Jiaotong University, \\ $X i$ ' an 710049, China \\ E-mail: huaifan.li@stu.xjtu.edu.cn,cairg@itp.ac.cn,hqzhang@itp.ac.cn
}

\begin{abstract}
We use the variational method for the Sturm-Liouville eigenvalue problem to analytically calculate some properties of holographic superconductors with GaussBonnet gravity in probe limit. By studying the holographic p-wave and s-wave superconductors in $(3+1)$-dimensional boundary field theories, it is found that near the critical temperature, the critical exponent of the condensation is $1 / 2$ which is the universal value in mean-field theory. We also find that when Gauss-Bonnet coefficients grow bigger the operators on the boundary field theory will be harder to condense. These are in good agreement with the numerical results.
\end{abstract}

Keywords: Holographic Superconductor, Gauss-Bonnet Gravity. 


\section{Contents}

1. Introduction 1

2. Ricci Flat Gauss-Bonnet-AdS Black Holes 2

3. Holographic P-wave Superconductors 3

3.1 Basic setup 3

3.2 Relations between the critical temperature and charge density 4

3.3 Critical exponents and the condensation values 7

4. Holographic S-wave Superconductors 9

4.1 Basic setup 9

4.2 Relations between the critical temperature and charge density 10

4.3 Critical exponents and the condensation values 12

5. Conclusions 13

\section{Introduction}

The AdS/CFT correspondence [1, 2, 3] provides a theoretical method to understand strongly coupled field theories. Recently, it has been proposed that the AdS/CFT correspondence can also be used to describe superconductor phase transitions 《4, 河. Since the high $T_{c}$ superconductors are shown to be in the strong coupling regime, one expects that the holographic method could give some insights into the pairing mechanism in the high $T_{c}$ superconductors.

There have been lots of works studying various holographic superconductors, see review [6, 7], in which some effects such as scalar field mass, external magnetic field, and back reaction etc., have been discussed. Among those works, some universality is discovered. For instance, the critical exponents for the condensation near the critical temperature is found to be $1 / 2$ which is a universal value in mean-field theory [8]. However, this value of $1 / 2$ is mostly obtained by numerically solving the holographic systems and then fitting the data. The frequently asked question is how to calculate this $1 / 2$ from analytical study. In [9], the authors used the variational method for the 
Sturm-Liouville (S-L) eigenvalue problem ${ }^{1}$ to analytically calculate some properties of the holographic superconductors in a $(2+1)$-dimensional boundary field theory. They managed to obtain the critical exponent $1 / 2$ by perturbing the system near the critical temperature. Afterwards, the authors in 10 extended this S-L method to study the holographic s, p, d-wave superconductors in 3-dimensional boundary field theory. They found that this $1 / 2$ is a universal value for these models.

In this paper, we extended the S-L method to study holographic p-wave and swave superconductors in a $(4+1)$-dimensional bulk with Gauss-Bonnet gravity. It is not trivial to extend this analytical studies to $(4+1)$-dimensional case, because the dimension of space-time will affect the boundary behavior of the fields which coupled to gravity. And moreover it would further have an impact on the conformal dimension of the dual operators. For instance, in $(2+1)$-dimensional boundary field theory the charge density $\rho \propto T^{2}$ where $T$ is the temperature; However, in $(3+1)$-dimensional boundary field theory the relation is $\rho \propto T^{3}$. This discrepancy would make the analysis a little different from the one in [9]. We will see it explicitly in the following context. Besides, we also calculated the ratio between the critical temperature and the charge density for various Gauss-Bonnet couplings. The ratio was greatly consistent with the previous numerical results [11, 12]. We also found that the greater the Gauss-Bonnet coefficients were, the harder the condensations to form, which was also consistent with the previous conclusions [13, 14, 15]. Although the conformal dimensions of the dual operators on boundary theory are different from those in [9], there still exists a universal critical exponents $1 / 2$ as in the mean-field theory. The ratios between the condensation values and the critical temperatures matched the numerical results up to the same order.

The paper is organized as follows: In Sec.(2) we briefly review the basic facts of Ricci flat Gauss-Bonnet-AdS black holes. In Sec.(3), we will analytically calculate the ratios between critical temperature and the charge density as well as the critical exponents of holographic p-wave superconductors. The same procedure will be followed in Sec.(4) by studying holographic s-wave superconductors. We will draw a conclusion in Sec.(5).

\section{Ricci Flat Gauss-Bonnet-AdS Black Holes}

The action of Gauss-Bonnet gravity in a 5-dimensional space-time can be written as

$$
S=\int d^{5} x \sqrt{-g}\left(R+\frac{12}{L^{2}}+\frac{\alpha}{2}\left(R^{2}-4 R^{\mu \nu} R_{\mu \nu}+R^{\mu \nu \rho \sigma} R_{\mu \nu \rho \sigma}\right)\right)
$$

\footnotetext{
${ }^{1}$ For short, we will call this method used by [9] to be "S-L method" in the following context.
} 
where $12 / L^{2}$ is related to the cosmological constant and $L$ is the radius of the AdS space-time. The quadratic curvature term in this action is the Gauss-Bonnet term with $\alpha$ the Gauss-Bonnet coefficient. The Ricci flat solution of this action is the 5dimensional Gauss-Bonnet-AdS black hole [16]. The metric is described by

$$
\begin{aligned}
& d s^{2}=-f(r) d t^{2}+\frac{1}{f(r)} d r^{2}+\frac{r^{2}}{L^{2}}\left(d x^{2}+d y^{2}+d z^{2}\right), \\
& f(r)=\frac{r^{2}}{2 \alpha}\left(1-\sqrt{1-\frac{4 \alpha}{L^{2}}\left(1-\frac{M L^{2}}{r^{4}}\right)}\right)
\end{aligned}
$$

where $M$ is the mass of the black hole. The horizon is located at $r=r_{+}=\sqrt[4]{M L^{2}}$, and the temperature of the black hole is

$$
T=\frac{r_{+}}{\pi L^{2}}
$$

Here we should notice that in the asymptotic region with $r \rightarrow \infty$,

$$
f(r) \sim \frac{r^{2}}{2 \alpha}\left[1-\sqrt{1-\frac{4 \alpha}{L^{2}}}\right] .
$$

Therefore, one can define an effective radius $L_{\text {eff }}$ of the AdS spacetime by

$$
L_{\mathrm{eff}}^{2} \equiv \frac{2 \alpha}{1-\sqrt{1-\frac{4 \alpha}{L^{2}}}} .
$$

We can see from this equation that one has to have $\alpha \leq L^{2} / 4$ in order to have a well-defined vacuum for the gravity theory. The upper bound $\alpha=L^{2} / 4$ is called Chern-Simons limit. In the AdS/CFT correspondence, this asymptotic AdS spacetime is dual to a conformal field theory living on the boundary $r \rightarrow \infty$. The temperature of the black hole is just the one of the dual field theory. For simplicity of making integration and making comparison to the previous numerical results, we will assume $0<\alpha \leq L^{2} / 5$ in the following context.

\section{Holographic P-wave Superconductors}

\subsection{Basic setup}

In order to study the holographic p-wave superconductors in the probe limit, we will introduce an SU(2) Yang-Mills action in to the bulk theory, the Lagrangian density is

$$
\mathscr{L}_{p}=-\frac{1}{4} F_{\mu \nu}^{a} F^{a \mu \nu}
$$


where, $F_{\mu \nu}^{a}=\partial_{\mu} A_{\nu}^{a}-\partial_{\nu} A_{\mu}^{a}+\epsilon^{a b c} A_{\mu}^{b} A_{\nu}^{c}$ is the Yang-Mills field strength, $a, b, c=(1,2,3)$ are the indices of the generators of $\mathrm{SU}(2)$ algebra. $\mu, \nu=(t, r, x, y, z)$ are the labels of space-time with $r$ being the radial coordinate of AdS. The $A_{\mu}^{a}$ are the components of the mixed-valued gauge fields $A=A_{\mu}^{a} \tau^{a} d x^{\mu}$, where $\tau^{a}$ are the $\mathrm{SU}(2)$ generators with commutation relation $\left[\tau^{a}, \tau^{b}\right]=\epsilon^{a b c} \tau^{c}$. $\epsilon^{a b c}$ is the totally antisymmetric tensor with $\epsilon^{123}=+1$.

Following Refs. [17, 18, 19, 20, 21], we choose the ansatz of the gauge fields as

$$
A(r)=\phi(r) \tau^{3} d t+\psi(r) \tau^{1} d x
$$

In this ansatz we regard the $\mathrm{U}(1)$ symmetry generated by $\tau^{3}$ as the $\mathrm{U}(1)$ subgroup of $\mathrm{SU}(2)$. We call this $\mathrm{U}(1)$ subgroup as $\mathrm{U}(1)_{3}$. The gauge boson with nonzero component $\psi(r)$ along $x$ direction is charged under $A_{t}^{3}=\phi(r)$. According to AdS/CFT dictionary, $\phi(r)$ is dual to the chemical potential in the boundary field theory while $\psi(r)$ is dual to the $x$ component of some charged vector operator $J$. The condensation of $\psi(r)$ will spontaneously break the $\mathrm{U}(1)_{3}$ gauge symmetry and induce the phenomena of superconducting on the boundary field theory.

The Yang-Mills equations with the above ansatz (3.2) are

$$
\left\{\begin{array}{l}
\partial_{r}^{2} \phi+\frac{3}{r} \partial_{r} \phi-\frac{L^{2} \psi^{2}}{r^{2} f} \phi=0, \\
\partial_{r}^{2} \psi+\left(\frac{1}{r}+\frac{\partial_{r} f}{f}\right) \partial_{r} \psi+\frac{\phi^{2}}{f^{2}} \psi=0 .
\end{array}\right.
$$

In order to solve the equations (3.3), we need the boundary conditions for the fields $\psi(r)$ and $\phi(r)$. At the black hole horizon, it is required that $\phi\left(r_{+}\right)=0$ for the $\mathrm{U}(1)$ gauge field to have a finite norm, and $\psi\left(r_{+}\right)$should be finite. Near the boundary of the bulk, we have

$$
\begin{aligned}
\phi(r) & \rightarrow \mu-\rho / r^{2} \\
\psi(r) & \rightarrow \psi^{(0)}+\psi^{(2)} / r^{2} .
\end{aligned}
$$

$\mu$ and $\rho$ are dual to the chemical potential and charge density of the boundary CFT , $\psi^{(0)}$ and $\psi^{(2)}$ are dual to the source and expectation value of the boundary operator $J_{x}^{1}$ respectively. We always set the source $\psi^{(0)}$ to zero, as we want to have a normalizable solution.

\subsection{Relations between the critical temperature and charge density}

In this subsection, we will adopt S-L method, which was pioneered by [9], to analytically calculate the ratios between the critical temperature $T_{c}$ and the charge density $\rho$ of the holographic p-wave superconductors. 
Define $z=\frac{r_{+}}{r}$, the EoMs $(3.3)$ will become

$$
\left\{\begin{array}{c}
\phi^{\prime \prime}-\frac{\phi^{\prime}}{z}-\frac{L^{2} \psi^{2}}{z^{2} f} \phi=0 \\
\psi^{\prime \prime}+\left(\frac{1}{z}+\frac{f^{\prime}}{f}\right) \psi^{\prime}+\frac{\phi^{2} r_{+}^{2}}{f^{2} z^{4}} \psi=0 .
\end{array}\right.
$$

where a prime denotes the derivative with respect to $z$. In the following we will scale $L=1$. When the temperature approaches the critical temperature i.e., $T \rightarrow T_{c}$, the condensation approaches zero, viz. $\psi \rightarrow 0$, from eq.(3.6) we can get

$$
\phi^{\prime \prime}-\frac{\phi^{\prime}}{z} \approx 0
$$

With the boundary conditions (3.4), we can get that near the critical temperature, the electric field behaves like

$$
\phi(z) \approx \lambda r_{+}\left(1-z^{2}\right)
$$

where, $\lambda=\rho / r_{+}^{3}$. Near the boundary, we will introduce a trial function $F(z)$ into $\psi(z)$ as in 9

$$
\left.\psi\right|_{z \rightarrow 0} \sim \psi^{(2)} \frac{z^{2}}{r_{+}^{2}} \sim\left\langle J_{x}^{1}\right\rangle \frac{z^{2}}{r_{+}^{2}} F(z) .
$$

where, $F(0)=1, F^{\prime}(0)=0$. Therefore, using (3.8) and (3.9) the EoM of $\psi(z)$ reduces to

$$
\begin{aligned}
F^{\prime \prime}(z) & +\frac{3+4 \alpha\left(5 z^{4}-3\right)-3 \sqrt{1+4 \alpha\left(z^{4}-1\right)}}{\left(1+4 \alpha\left(z^{4}-1\right)-\sqrt{1+4 \alpha\left(z^{4}-1\right)}\right) z} F^{\prime}(z) \\
& +\frac{16 \alpha z^{2}}{1+4 \alpha\left(z^{4}-1\right)-\sqrt{1+4 \alpha\left(z^{4}-1\right)}} F(z)+\lambda^{2} \frac{4 \alpha^{2}\left(z^{2}-1\right)^{2}}{\left(\sqrt{1+4 \alpha\left(z^{4}-1\right)}-1\right)^{2}} F(z)=0 .
\end{aligned}
$$

Multiplying both sides of the above equation with

$$
T(z)=\frac{z^{3}}{2 \sqrt{\alpha}}\left(\sqrt{1+4 \alpha\left(z^{4}-1\right)}-1\right),
$$

We will convert the eq. (3.10) to be

$$
\frac{d}{d z}\left(T(z) F^{\prime}(z)\right)+\underbrace{\frac{8 \sqrt{\alpha} z^{5}}{\sqrt{1+4 \alpha\left(z^{4}-1\right)}}}_{-Q(z)} F(z)+\lambda^{2} \underbrace{\left(\frac{4 \alpha^{2}\left(z^{2}-1\right)^{2} T(z)}{\left(\sqrt{1+4 \alpha\left(z^{4}-1\right)}-1\right)^{2}}\right)}_{P(z)} F(z)=0 .
$$


From the Sturm-Liouville eigenvlaue problem [22], the minimum of eigenvalues of $\lambda^{2}$ can be obtained from the variation of the following functional

$$
\lambda^{2}[F(z)]=\frac{\int_{0}^{1} d z\left(T(z) F^{\prime}(z)^{2}+Q(z) F(z)^{2}\right)}{\int_{0}^{1} d z P(z) F(z)^{2}},
$$

The trial function $F(z)$ can be assumed to be

$$
F(z) \equiv 1-a z^{2}
$$

which satisfies the boundary condition. So $\lambda^{2}(z)$ can be explicitly written as

$$
\lambda^{2}(z)=\frac{s(a, \alpha)}{t(a, \alpha)} .
$$

where,

$$
\begin{aligned}
s(a, \alpha)= & 60(4 \alpha-1)\left((4 \alpha-1) a^{2}+4 \alpha\right) \log \left(\frac{(1-4 \alpha) \alpha}{(2 \alpha+\sqrt{\alpha})^{2}}\right) \\
& -80 \sqrt{\alpha}(12 \alpha+a(20 \alpha a-3 a+32 \sqrt{1-4 \alpha} \alpha-48 \alpha-8 \sqrt{1-4 \alpha}+8)) \\
t(a, \alpha)= & \frac{15}{2}(a+1) \alpha\left[128(a+1) \log \left(\frac{-4 \alpha+\sqrt{1-4 \alpha}+1}{2-8 \alpha}\right) \alpha^{3 / 2}\right. \\
& \left.+\left(16(3 a+4) \alpha^{2}+8(3 a+2) \alpha-a\right) \log \left(\frac{(1-4 \alpha) \alpha}{(2 \alpha+\sqrt{\alpha})^{2}}\right)\right] \\
& -2 \sqrt{\alpha}\left((\alpha(32 \alpha(9 \sqrt{1-4 \alpha}-30 \log 2+5)+56 \sqrt{1-4 \alpha}-45)-2 \sqrt{1-4 \alpha}+2) a^{2}\right. \\
& +5 \alpha(4 \alpha(32 \sqrt{1-4 \alpha}-96 \log 2+11)+16 \sqrt{1-4 \alpha}-13) a \\
& +20 \alpha(\alpha(20 \sqrt{1-4 \alpha}-48 \log 2+6)+\sqrt{1-4 \alpha}-1))
\end{aligned}
$$

For different values of $\alpha$, we list the minimum value of $\lambda^{2}$ and the corresponding value of $a$ in Table(1). Because $T=r_{+} / \pi$ and $\lambda=\rho / r_{+}^{3}$, we can easily get that when $T \sim T_{c}$, there is

$$
T_{c} \approx \gamma \sqrt[3]{\rho}
$$

where, $\gamma=1 /\left(\pi \lambda_{\text {min }}^{1 / 3}\right)$.

In Table(1), we also list the analytical values and numerical values [12] of $\gamma$ for different $\alpha$. The differences between the analytical and numerical values are within 
Table 1: The minimum values of $\lambda^{2}$ and the corresponding $a$ for different $\alpha$ are listed in the first three columns. The analytical and numerical values of $\gamma$ and $\zeta$ are shown in the last four columns. The differences between the analytical and numerical values of $\gamma$ is about $1 \%$ for various $\alpha$ 's. The order for $\zeta_{\text {analytical }}$ and $\zeta_{\text {numerical }}$ is the same.

\begin{tabular}{|c|c|c||c|c||c|c|}
\hline$\alpha$ & $a$ & $\lambda_{\text {min }}^{2}$ & $\gamma_{\text {analytical }}$ & $\gamma_{\text {numerical }}$ & $\zeta_{\text {analytical }}$ & $\zeta_{\text {numerical }}$ \\
\hline \hline 0.0001 & 0.685 & 16.748 & 0.199 & 0.201 & 326.858 & 499.036 \\
\hline 0.1 & 0.677 & 20.604 & 0.192 & 0.194 & 337.735 & 509.796 \\
\hline 0.2 & 0.653 & 28.571 & 0.182 & 0.183 & 349.614 & 519.583 \\
\hline
\end{tabular}

1\%. We can find that when $\alpha$ grows, the ratio $\gamma$ will decrease, which means the critical temperature will get lower compared to the charge density. This implies that the greater the Gauss-Bonnet coefficient is, the harder the condensation to form. This statement is consistent with the previous numerical results [11, 12, 13, 14].

\subsection{Critical exponents and the condensation values}

From last subsection we know that when $T \rightarrow T_{c}$, the condensation value of the dual operator $\left\langle J_{x}^{1}\right\rangle$ is very small. Using formula (3.9) we can convert the eq.(3.6) to be

$$
\phi^{\prime \prime}-\frac{\phi^{\prime}}{z}=\frac{\mathcal{A} z^{2} F^{2}}{f} \phi,
$$

where, $\mathcal{A}=\left\langle J_{x}^{1}\right\rangle^{2} / r_{+}^{4}$. Because $\mathcal{A}$ is small, considering (3.8) we can expand $\phi(z)$ in $\mathcal{A}$ as

$$
\frac{\phi}{r_{+}} \sim \lambda\left(1-z^{2}\right)+\mathcal{A} \chi(z)+\cdots,
$$

From the boundary condition we can get $\chi(1)=0$, and for simplicity we set $\chi^{\prime}(1)=0$ [9]. The eq.(3.18) now becomes

$$
\chi^{\prime \prime}-\frac{\chi^{\prime}}{z}=\lambda \frac{z^{2} F^{2}\left(1-z^{2}\right)}{f} .
$$

When temperature is away but very close to the critical temperature, we expand $\phi(z)$ near $z=0$,

$$
\begin{aligned}
\phi=\mu-\frac{\rho}{r_{+}^{2}} z^{2}=\frac{\rho}{r_{+}^{2}}\left(1-z^{2}\right) \Rightarrow \frac{\phi}{r_{+}} & =\frac{\rho}{r_{+}^{3}}\left(1-z^{2}\right) \sim \lambda\left(1-z^{2}\right)+\mathcal{A} \chi(z)+\cdots \\
& =\lambda\left(1-z^{2}\right)+\mathcal{A}\left(\chi(0)+\chi^{\prime}(0) z+\frac{\chi^{\prime \prime}(0)}{2} z^{2}+\cdots\right)
\end{aligned}
$$


Comparing the coefficients of $z^{2}$ term in the above formula ${ }^{2}$ we can get

$$
\frac{\rho}{r_{+}^{3}}=\lambda-\frac{\mathcal{A}}{2} \chi^{\prime \prime}(0)
$$

From the eq.(3.20) we know that

$$
\chi^{\prime \prime}(0)=\left.\left[\frac{\chi^{\prime}(z)}{z}+\frac{\mathcal{A} z^{2} F^{2}}{f} \phi\right]\right|_{z \rightarrow 0}=\left.\left[\frac{\chi^{\prime}(z)}{z}\right]\right|_{z \rightarrow 0}
$$

Because the second term in the r.h.s of the first equality approaches zero when $z \rightarrow 0$. In order to calculate the value of $\left.\left[\frac{\chi^{\prime}(z)}{z}\right]\right|_{z \rightarrow 0}$, fortunately we find that multiply $1 / z$ to both sides of eq.(3.20) we get

$$
\left(\frac{1}{z} \chi^{\prime}\right)^{\prime}=\lambda \frac{z F^{2}\left(1-z^{2}\right)}{f}
$$

Integrate both sides of the above equation and note that $\chi^{\prime}(1)=0$, we obtain ${ }^{3}$

$$
\left.\left[\frac{\chi^{\prime}(z)}{z}\right]\right|_{z \rightarrow 0}=-\lambda \int_{0}^{1} d z \frac{z F^{2}\left(1-z^{2}\right)}{f}
$$

Therefore, from (3.22) one has

$$
\frac{\rho}{r_{+}^{3}}=\lambda+\frac{\mathcal{A}}{2} \lambda \int_{0}^{1} d z \frac{z F^{2}\left(1-z^{2}\right)}{f}
$$

From the form of $\mathcal{A}$ and $f(z)$ we can obtain

$$
\frac{\rho}{\lambda r_{+}^{3}}=1+\frac{\mathcal{B}\left\langle J_{x}^{1}\right\rangle^{2}}{2 r_{+}^{6}}
$$

where $\mathcal{B}=\int_{0}^{1} d z\left(z F^{2}(z)\left(1-z^{2}\right) / g(z)\right)$ and $g(z)=f(z) / r_{+}^{2}$. So we can deduce that

$$
\left\langle J_{x}^{1}\right\rangle=\zeta T_{c}^{3} \sqrt{1-\frac{T}{T_{c}}} .
$$

where $\zeta=\sqrt{6} \pi^{3} / \sqrt{\mathcal{B}}$.

\footnotetext{
${ }^{2}$ Here, the analysis is a little different from [9] in which they compared the coefficients of $z$ term. The main difference comes from the near boundary behavior of $\phi(z)$ which deeply originates from the dimension of space-time.

${ }^{3}$ Comparing the $z$ term in (3.21), we know that $\chi^{\prime}(0) \rightarrow 0$ when $z \rightarrow 0$. This is consistent with (3.25).
} 
The analytical values and numerical values [12] of $\zeta$ for various $\alpha$ 's can be found in Table.(1). They match up to the same order. Besides, in both analytical and numerical cases, the ratio $\zeta$ increases when $\alpha$ grows bigger, which implies that the condensation values for the dual operators will also increase. From the AdS/CFT dictionary, this operator can be interpreted as the operator for the paring mechanism, the bigger vacuum expectation value of this operator will make the condensation harder. This statement is also in agreement with the previous arguments [11, 12, 13, 14]. .

\section{Holographic S-wave Superconductors}

\subsection{Basic setup}

In order to study the holographic s-wave superconductors, we consider the Maxwell field couples to a complex scalar field in the bulk. The Lagrangian density is [5],

$$
\mathscr{L}_{s}=-\frac{1}{4} F_{\mu \nu} F^{\mu \nu}-|\nabla \psi-i A \psi|^{2}-m^{2}|\psi|^{2}
$$

where $A_{\mu}(r)$ is the vector field and $m^{2}$ is the mass square of the scalar field. As usual, we can choose the gauge of vector field as $A_{\mu}=(\phi(r), 0,0,0)$ which is consistent with the ansatz of a real scalar field $\psi(r)=\psi(r)^{*}$. In the following, we also scale the AdS radius to be $L=1$.

The EoMs for $\phi(r)$ and $\psi(r)$ are

$$
\left\{\begin{array}{c}
\partial_{r}^{2} \phi+\frac{3}{r} \partial_{r} \phi-\frac{2 \psi^{2}}{f} \phi=0 \\
\partial_{r}^{2} \psi+\left(\frac{\partial_{r} f}{f}+\frac{3}{r}\right) \partial_{r} \psi+\left(\frac{\phi^{2}}{f^{2}}-\frac{m^{2}}{f}\right) \psi=0 .
\end{array}\right.
$$

Near the boundary, $\phi(r)$ and $\psi(r)$ behave like

$$
\begin{aligned}
& \phi(r)=\mu-\frac{\rho}{r^{2}}, \\
& \psi(r)=\frac{\psi^{-}}{r^{\Delta_{-}}}+\frac{\psi^{+}}{r^{\Delta_{+}}} .
\end{aligned}
$$

where, $\mu$ and $\rho$ have the same interpretation as those in Sec.(3) $. \Delta_{ \pm}=2 \pm \sqrt{4+m^{2} L_{\text {eff }}^{2}}$ is the conformal dimension of the dual operator $J$ in the boundary field theory. We will also set $\psi^{-}=0$ and $\langle J\rangle=\psi^{+}$. Therefore, we will unambiguously define $\Delta \equiv \Delta_{+}$ from now on. On the horizon, $\phi(r)=0$ because of the well definition of the norm. 


\subsection{Relations between the critical temperature and charge density}

Then, we can define $z=r_{+} / r$, the EoMs (4.2) become

$$
\left\{\begin{array}{c}
\phi^{\prime \prime}-\frac{1}{z} \phi^{\prime}-\frac{2 \psi^{2} r_{+}^{2}}{f z^{4}} \phi=0, \\
\psi^{\prime \prime}+\left(\frac{f^{\prime}}{f}-\frac{1}{z}\right) \psi^{\prime}+\left(\frac{\phi^{2}}{f^{2}}-\frac{m^{2}}{f}\right) \frac{r_{+}^{2}}{z^{4}} \psi=0 .
\end{array}\right.
$$

where a prime denotes the derivative with respect to $z$.

Because when $T \rightarrow T_{c}$, the condensation is very small, $\psi \sim 0$. Therefore, the EoM of $\phi(z)$ becomes

$$
\phi^{\prime \prime}-\frac{1}{z} \phi^{\prime}=0 .
$$

With the boundary condition (4.3), we can deduce that near the critical temperature $\phi(z) \sim \lambda r_{+}\left(1-z^{2}\right)$ where $\lambda \equiv \rho / r_{+}^{3}$.

As in the last section, we can introduce a trial function $F(z)$ into the asymptotical behavior of $\psi(z)$,

$$
\left.\psi\right|_{z \rightarrow 0} \sim \frac{\langle J\rangle}{r_{+}^{\Delta}} z^{\Delta} F(z) .
$$

where $F(0)=1, F^{\prime}(0)=0$. Now, the EoM for scalar field reduces to

$$
F^{\prime \prime}(z)+p(z) F^{\prime}(z)+q(z) F(z)+\lambda^{2} w(z) F(z)=0 .
$$

where,

$$
\begin{aligned}
p(z)= & \frac{\left(1-\sqrt{4\left(z^{4}-1\right) \alpha+1}\right)(2 \Delta-3)+4 \alpha\left((2 \Delta-1) z^{4}-2 \Delta+3\right)}{z\left(4\left(z^{4}-1\right) \alpha-\sqrt{4\left(z^{4}-1\right) \alpha+1}+1\right)} \\
q(z)= & \frac{1}{z}\left[-\frac{\left(3\left(\sqrt{4\left(z^{4}-1\right) \alpha+1}-1\right)-4\left(z^{4}-3\right) \alpha\right) \Delta}{-4\left(z^{4}-1\right) \alpha+\sqrt{4\left(z^{4}-1\right) \alpha+1}-1}\right. \\
& \left.+\frac{2 m^{2} \alpha}{\sqrt{4\left(z^{4}-1\right) \alpha+1}-1}+(\Delta-1) \Delta\right] \\
w(z)= & \frac{4\left(z^{2}-1\right)^{2} \alpha^{2}}{\left(\sqrt{4\left(z^{4}-1\right) \alpha+1}-1\right)^{2}} .
\end{aligned}
$$

Multiply both sides of (4.8) with

$$
T(z)=\frac{z^{2 \Delta-3}}{2 \sqrt{\alpha}}\left(\sqrt{1+4 \alpha\left(z^{4}-1\right)}-1\right),
$$


we obtain the following equation

$$
\left(T(z) F^{\prime}(z)\right)^{\prime}-Q(z) F(z)+\lambda^{2} P(z) F(z)=0
$$

where

$$
\begin{aligned}
& Q(z)=-T(z) q(z) \\
& P(z)=T(z) w(z)
\end{aligned}
$$

Following the procedure used in the above section, we know that the minimum value of $\lambda^{2}$ can be obtained from variation of the following functional

$$
\lambda^{2}[F(z)]=\frac{\int_{0}^{1} d z\left(T(z) F^{\prime}(z)^{2}+Q(z) F(z)^{2}\right)}{\int_{0}^{1} d z P(z) F(z)^{2}},
$$

It is easy to find that $F(z)=1-a z^{2}$ and in the calculation we will assume $m^{2}=-3 / L_{\text {eff }}^{2}$ in order to make comparison with the previous results [11]. So $\lambda^{2}(z)$ can be explicitly written as

$$
\lambda^{2}(z)=\frac{s(a, \alpha)}{t(a, \alpha)}
$$

where,

$$
\begin{aligned}
s(a, \alpha)= & -\frac{1}{768 \alpha^{2}}\left[2 \sqrt { \alpha } \left((96 \sqrt{1-4 \alpha} \alpha+404 \alpha-75) a^{2}\right.\right. \\
& +48(14 \sqrt{1-4 \alpha} \alpha-24 \alpha-5 \sqrt{1-4 \alpha}+5) a+144(2 \sqrt{1-4 \alpha} \alpha+\alpha)) \\
& \left.+3(4 \alpha-1)\left(25(4 \alpha-1) a^{2}+144 \alpha\right) \sinh ^{-1}\left(2 \sqrt{\frac{\alpha}{1-4 \alpha}}\right)\right] \\
t(a, \alpha)= & \frac{\sqrt{1-4 \alpha}}{480 \alpha^{3 / 2}}\left(\left(-144 \alpha^{2}-28 \alpha+1\right) a^{2}-40 \alpha(8 \alpha+1) a-10 \alpha(20 \alpha+1)\right) \\
& +\frac{1}{2}(a+1)^{2} \sqrt{\alpha} \log (-4 \alpha+\sqrt{1-4 \alpha}+1) \\
& +\frac{(a+1)\left(16 \alpha(4 \alpha+1)+a\left(48 \alpha^{2}+24 \alpha-1\right)\right) \log (\sqrt{1-4 \alpha} \sqrt{\alpha})}{128 \alpha} \\
& +\frac{1}{1920 \alpha^{3 / 2}}\left[-960(a+1)^{2} \log (2-8 \alpha) \alpha^{2}\right. \\
& -15(a+1)\left(16 \alpha(4 \alpha+1)+a\left(48 \alpha^{2}+24 \alpha-1\right)\right) \log (2 \alpha+\sqrt{\alpha}) \sqrt{\alpha} \\
& +\left(920 \alpha^{2}+90 \alpha-4\right) a^{2}+10 \alpha(212 \alpha+13) a+960(a+1)^{2} \log (2) \alpha^{2} \\
& \left.+40(24 \alpha+1) \alpha-960(a+1)^{2} \alpha^{2} \Gamma^{\prime}\left(\frac{3}{2}\right) / \Gamma\left(\frac{3}{2}\right)-960(a+1)^{2} \gamma_{\operatorname{Euler}} \alpha^{2}\right] \quad(4.19)
\end{aligned}
$$


Table 2: For $m^{2}=-3 / L_{\text {eff }}^{2}$, the minimum values of $\lambda^{2}$ and the corresponding $a$ for various $\alpha$ 's are listed in the first three columns. The analytical values from S-L approach are very similar to those obtained from matching methods [11] with differences smaller than $4 \%$. The values of $\zeta$ 's are matching up to the same order.

\begin{tabular}{|c|c|c||c|c||c|c|}
\hline$\alpha$ & $a$ & $\lambda_{\text {min }}^{2}$ & $\gamma_{\mathrm{S}-\mathrm{L}}$ & $\gamma_{\text {matching }}$ & $\zeta_{\mathrm{S}-\mathrm{L}}$ & $\zeta_{\text {matching }}$ \\
\hline \hline 0.0001 & 0.722 & 12.155 & 0.210 & 0.202 & 7.705 & 6.369 \\
\hline 0.1 & 0.706 & 14.290 & 0.204 & 0.197 & 7.913 & 6.884 \\
\hline 0.2 & 0.662 & 18.091 & 0.196 & 0.193 & 8.042 & 7.596 \\
\hline
\end{tabular}

in which, $\Gamma(z)$ is the gamma function and $\gamma_{\text {Euler }}$ is the Euler's constant. Because $T=r_{+} / \pi$ and $\lambda=\rho / r_{+}^{3}$, we can easily get that when $T \sim T_{c}$, there is

$$
T_{c}=\gamma \sqrt[3]{\rho}
$$

where, $\gamma=1 /\left(\pi \lambda_{\text {min }}^{1 / 3}\right)$.

We list the analytical results in Table(2). We can find that the values of $\gamma$ obtained from the S-L approach are very close to those calculated in [11] by the matching methods ${ }^{4}$. The differences are smaller than $4 \%$ for various $\alpha$ 's. We also find that $\gamma$ decreases when $\alpha$ grows implying that the condensation is harder to form, which is consistent with the previous results [11, 13.

\subsection{Critical exponents and the condensation values}

When $T \rightarrow T_{c}$, from the equation of $\phi(z)$, we get

$$
\phi^{\prime \prime}-\frac{1}{z} \phi^{\prime}=\frac{2 \mathcal{A} \phi z^{2 \Delta-4} F^{2}}{g}
$$

where, $\mathcal{A} \equiv\langle J\rangle^{2} / r_{+}^{2 \Delta}, g(z)=f(z) / r_{+}^{2}$. Because near the critical temperature $T_{c}, \mathcal{A}$ is small. We can expand $\phi(z)$ in $\mathcal{A}$ near $z=0$,

$$
\frac{\phi}{r_{+}}=\lambda\left(1-z^{2}\right)+\mathcal{A} \chi(z)+\cdots
$$

Comparing the coefficients in $z^{2}$ terms, we can get from the above formula that

$$
\frac{\rho}{r_{+}^{3}}=\lambda-\frac{\mathcal{A}}{2} \chi^{\prime \prime}(0)
$$

Substituting (4.22) into eq.(4.21), we can get EoM for $\chi(z)$ as

$$
\chi^{\prime \prime}-\frac{1}{z} \chi^{\prime}=2 \lambda \frac{z^{2 \Delta-4} F^{2}\left(1-z^{2}\right)}{g}
$$

\footnotetext{
${ }^{4}$ Consult Eq. (3.27) in Ref.[1].
} 
Multiplying $1 / z$ to both sides and following the procedure in last section, we obtain

$$
\chi^{\prime \prime}(0)=\left.\frac{\chi^{\prime}(z)}{z}\right|_{z \rightarrow 0}=-2 \lambda \int_{0}^{1} d z \frac{z^{2 \Delta-5} F^{2}\left(1-z^{2}\right)}{g} .
$$

Therefore, (4.23) becomes

$$
\frac{\rho}{r_{+}^{3}}=\lambda\left(1+\frac{\mathcal{A}}{2} \mathcal{B}\right)
$$

where $\mathcal{B}=2 \int_{0}^{1} d z z^{2 \Delta-5} F^{2}\left(1-z^{2}\right) / g$.

Finally, using the formula of $\mathcal{A}$ we get that when $T \rightarrow T_{c}$, the condensation of the operator $J$ behaves like

$$
\langle J\rangle=\zeta\left(\pi T_{c}\right)^{\Delta} \sqrt{1-\frac{T}{T_{c}}}
$$

where, $\zeta=\sqrt{6} / \sqrt{\mathcal{B}}$. In Table(2) we list the value of $\zeta$ in S-L method and those in matching method $[11]^{5}$. We find that these two kinds of values are matching up to the same order. The ratio $\zeta$ increases when $\alpha$ grows bigger, which implies that the condensation will be harder to form. This is consistent with the previous results [11, 13].

\section{Conclusions}

In this paper, we have used the S-L method to analytically calculate the holographic p-wave and s-wave superconductors with Gauss-Bonnet gravity in the probe limit. In particular, we calculate the ratios $\gamma$ between the critical temperature and the charge density on the boundary. The ratios are in great agreement with the previous numerical results or those obtained from the analytical matching methods. We find that the ratio will decrease when the Gauss-Bonnet couplings $\alpha$ increases, which implies that the bigger $\alpha$ is the harder the condensation to form. This statement is in agreement with the previous conclusions. Besides, we also calculate the ratio $\zeta$ between the condensation value and the critical temperature. Although the values of ratio $\zeta$ is not so well agreement with the numerical results or those from matching methods, they are consistent up to the same order. Moreover, we find that when $\alpha$ increases, the ratio $\zeta$ will also increase. This again implies that the bigger $\alpha$ is the harder the condensation to form, which also agrees with the previous conclusions. The universal critical exponents $1 / 2$ in mean-field theory was also analytically confirmed in both p-wave and s-wave case of Gauss-Bonnet gravity. This critical exponents will not be

\footnotetext{
${ }^{5}$ Refer to Eq. (3.28) in Ref. 11
} 
affected by the dimension of the space-time or the Gauss-Bonnet coefficients $\alpha$. This value $1 / 2$ is an exact property of the Lagrangian for the matter field of systems, and has nothing to do with the gravitational backgrounds [23].

\section{Acknowledgments}

HFL and HQZ would like to thank Xin Gao, Bin Hu, Zhang-Yu Nie and Yuan-Jiang Zhang for their helpful discussions and comments. HFL would be very grateful for the hospitalities of the members in the Institute of Theoretical Physics, Chinese Academy of Sciences. This work was supported in part by the National Natural Science Foundation of China (No. 10821504, No. 10975168, No.11035008 and No.11075098), and in part by the Ministry of Science and Technology of China under Grant No. 2010CB833004.

\section{References}

[1] J. M. Maldacena, "The large N limit of superconformal field theories and supergravity," Adv. Theor. Math. Phys. 2, 231 (1998) [Int. J. Theor. Phys. 38, 1113 (1999)] [arXiv:hep-th/9711200].

[2] S. S. Gubser, I. R. Klebanov and A. M. Polyakov, "Gauge theory correlators from non-critical string theory," Phys. Lett. B 428, 105 (1998) [arXiv:hep-th/9802109].

[3] E. Witten, "Anti-de Sitter space and holography," Adv. Theor. Math. Phys. 2, 253 (1998) [arXiv:hep-th/9802150].

[4] S. S. Gubser, "Breaking an Abelian gauge symmetry near a black hole horizon," Phys. Rev. D 78, 065034 (2008) [arXiv:0801.2977 [hep-th]].

[5] S. A. Hartnoll, C. P. Herzog and G. T. Horowitz, "Building a Holographic

Superconductor," Phys. Rev. Lett. 101, 031601 (2008) [arXiv:0803.3295 [hep-th]].

[6] S. A. Hartnoll, "Lectures on holographic methods for condensed matter physics," Class. Quant. Grav. 26, 224002 (2009) [arXiv:0903.3246 [hep-th]].

[7] C. P. Herzog, "Lectures on Holographic Superfluidity and Superconductivity," J. Phys. A 42, 343001 (2009) [arXiv:0904.1975 [hep-th]].

[8] J. Bardeen, L. N. Cooper and J. R. Schrieffer, "Theory Of Superconductivity," Phys. Rev. 108 (1957) 1175.

[9] G. Siopsis and J. Therrien, "Analytic calculation of properties of holographic superconductors," JHEP 1005 (2010) 013 [arXiv:1003.4275 [hep-th]]. 
[10] H. B. Zeng, X. Gao, Y. Jiang and H. S. Zong, "Analytical Computation of Critical Exponents in Several Holographic Superconductors," arXiv:1012.5564 [hep-th].

[11] R. Gregory, S. Kanno and J. Soda, "Holographic Superconductors with Higher Curvature Corrections," JHEP 0910, 010 (2009) [arXiv:0907.3203 [hep-th]].

[12] R. G. Cai, Z. Y. Nie and H. Q. Zhang, "Holographic p-wave superconductors from Gauss-Bonnet gravity," Phys. Rev. D 82 (2010) 066007 [arXiv:1007.3321 [hep-th]].

[13] Q. Pan, B. Wang, E. Papantonopoulos, J. Oliveira and A. B. Pavan, "Holographic Superconductors with various condensates in Einstein-Gauss-Bonnet gravity," Phys. Rev. D 81, 106007 (2010) [arXiv:0912.2475 [hep-th]].

[14] R. G. Cai, Z. Y. Nie and H. Q. Zhang, "Holographic Phase Transitions of P-wave Superconductors in Gauss-Bonnet Gravity with Back-reaction," arXiv:1012.5559 [hep-th].

[15] Y. Brihaye and B. Hartmann, "Holographic Superconductors in 3+1 dimensions away from the probe limit," Phys. Rev. D 81, 126008 (2010) [arXiv:1003.5130 [hep-th]].

[16] R. G. Cai, "Gauss-Bonnet black holes in AdS spaces," Phys. Rev. D 65, 084014 (2002) [arXiv:hep-th/0109133].

[17] S. S. Gubser and S. S. Pufu, "The gravity dual of a p-wave superconductor," JHEP 0811, 033 (2008) [arXiv:0805.2960 [hep-th]].

[18] R. Manvelyan, E. Radu and D. H. Tchrakian, "New AdS non Abelian black holes with superconducting horizons," Phys. Lett. B 677, 79 (2009) [arXiv:0812.3531 [hep-th]].

[19] P. Basu, J. He, A. Mukherjee and H. H. Shieh, "Hard-gapped Holographic Superconductors," Phys. Lett. B 689, 45 (2010) [arXiv:0911.4999 [hep-th]].

[20] M. Ammon, J. Erdmenger, V. Grass, P. Kerner and A. O’Bannon, "On Holographic p-wave Superfluids with Back-reaction," Phys. Lett. B 686, 192 (2010) [arXiv:0912.3515 [hep-th]].

[21] A. Akhavan and M. Alishahiha, "P-Wave Holographic Insulator/Superconductor Phase Transition," arXiv:1011.6158 [hep-th].

[22] I. M. Gelfand and S. V. Fomin, "Calculaus of Variations," (1963) Revised English Edition, Translated and Edited by R. A. Silverman, Prentice-Hall, Inc. Englewood Cliff, New Jersey.

[23] Y. Liu, Q. Pan, B. Wang and R. G. Cai, "Dynamical perturbations and critical phenomena in Gauss-Bonnet-AdS black holes," Phys. Lett. B 693, 343 (2010) [arXiv:1007.2536 [hep-th]]. 